Psicologia Escolar

e Educacional
ARTIGO

DOI: http://dx.doi.org/10.1590/2175-35392020213295

Elocid - e213295

\title{
MENTORIA DO TALENTO NA ESCOLA: ANÁLISE DE PUBLICAÇÕES NACIONAIS E INTERNACIONAIS
}

\author{
Janete Tonete Suárez ${ }^{1} \mathbb{D}$; Solange Múglia Wechsler ${ }^{2} \mathbb{D}$ \\ RESUMO
}

Há evidências de que mentores fazem diferença na vida de estudantes talentosos/superdotados, motivo da investigação do tema e sua produção científica nacional e internacional de 2000 a 2018. Entre as bases de dados investigadas estão: Scientific Electronic Library Online (Scielo), Banco de Teses/Dissertações Capes, Academic Search Premier, Portal Periódicos Capes e Sage Journals. Foram encontrados 59 estudos, sendo 24 nacionais e 35 internacionais. Sobressaíram-se estudos qualitativos com ênfase na abordagem exploratória. Na amostragem, destacaram-se professores do Ensino Fundamental, Médio e Superior. Entre os objetivos, destacou-se o interesse dos pesquisadores pelo processo de formação de professores por meio da mentoria. Percebeu-se, ainda, que estratégias de mentoria são eficazes para estudantes talentosos, melhorando a compreensão de conceitos como os matemáticos, por exemplo, além de benefícios psicossociais e de carreira. Quanto aos instrumentos, estudos internacionais fazem uso dos mais específicos e maior variedade deles. O tema é inovador, o que justifica sua investigação.

Palavras-chave: desenvolvimento humano; aptidão; superdotados.

\section{School Talent Mentoring: Analysis of National and International Publications}

\section{ABSTRACT}

There is evidence that mentors make a difference in the lives of talented / gifted students, the reason for the investigation of the theme and their national and international scientific production from 2000 to 2018. Among the databases investigated are Scientific Electronic Library Online (Scielo), Capes Theses / Dissertations, Academic Search Premier, Capes Journal Portal and Sage Journals. We found 59 studies, 24 national and 35 international. Qualitative studies stood out with emphasis on the exploratory approach. In the sample, teachers of elementary, middle and higher education stood out. Among the objectives, the researchers' interest in the process of teacher education through mentoring was highlighted. Mentoring strategies were also found to be effective for talented students, improving understanding of concepts such as mathematicians, for example, as well as psychosocial and career benefits. As for the instruments, international studies make use of the most specific and the widest variety of them. The theme is innovative, which justifies its investigation.

Keywords: human development; ability; gifted.

\section{Tutoría del Talento en la Escuela: Análisis de Publicaciones Nacionales e Internacionales}

\section{RESUMEN}

Hay evidencias de que tutores hacen diferencia en la vida de estudiantes talentosos/superdotados, motivo de la investigación del tema y su producción científica nacional e internacional de 2000 a 2018 . Entre las bases de datos investigadas están: Scientific Electronic Library Online (Scielo), Banco de Tesis/Tesinas Capes, Academic Search Premier, Portal Periódicos Capes y Sage Journals. Se obtuvo 59 estudios, es decir, 24 nacionales y 35 internacionales. Sobresalieron los estudios cualitativos con énfasis en el abordaje exploratorio. En el muestreo, predominaron profesores de la Enseñanza Básica, Secundaria y Universitaria. Entre os objetivos, se destacó el interés de los investigadores por el proceso de formación de profesores por intermedio de la tutoría. Se percibió, aún, que estrategias de tutoría son eficaces para estudiantes talentosos, mejorando la comprensión de conceptos como los matemáticos, por ejemplo, además de beneficios psicosociales y de carrera. En lo que se refiere a os instrumentos, estudios internacionales hacen uso de los más específicos y más variedad de ellos. El tema es innovador, lo que justifica su investigación.

Palabras clave: desarrollo humano; aptitud; superdotados.

\footnotetext{
${ }^{1}$ Universidade de Brasília - Brasília - DF - Brasil; janete.suarez@yahoo.com.br

${ }^{2}$ Pontifícia Universidade Católica de Campinas - Campinas - SP - Brasil; wechsler@lexxa.com.br
} 


\section{INTRODUÇÃO}

A escola normalmente trabalha visando a educação da maioria dos estudantes. Consequentemente, para aqueles que possuem necessidades educacionais especiais, como altas habilidades/superdotação, o atendimento profissional é raro, precário ou inexistente (Freeman, 2001; Shaughnessy, 2005). O conceito de "talento", utilizado neste estudo, está relacionado ao alto desempenho, domínio superior, mestria em habilidades e competências desenvolvidas de maneira sistemática pelo menos em um campo de ação. A preferência pelo termo ocorre por seu uso prático e que melhor instiga o diálogo sobre o assunto no contexto educacional. Esse termo dispensa a ideia de elitismo, ou seja, não privilegia apenas estudantes com desempenho superior, mas inclui pelo menos $10 \%$ ou mais dos melhores nas mais diversas áreas de atividade humana, considerando a mesma faixa etária e atividade (Gagné \& Güenther, 2012).

Quanto à identificação de estudantes talentosos, Tourón, Peralta e Repáraz (1998) acreditam que é mais valioso e útil reconhecer os pontos fortes de um talento específico do que simplesmente dizer que uma criança é superdotada. Na mesma direção, outros autores acreditam que não basta ser talentoso, é preciso contar com um contexto adequado de ensino e aprendizagem que potencie o talento e crie condições favoráveis para que estes estudantes se tornem futuros líderes em seus campos de interesse (Zorman, Rachmel, \& Barshan, 2016).

Um segundo termo que descreve essa população é "superdotação", o qual deve ser usado com cautela pelo risco de passar a ideia exagerada e falsa de um comportamento típico apenas de pessoas com potencial bastante elevado, constituindo um fenômeno raro (Gagné \& Güenther, 2012). Superdotação, adotado pela Política Nacional de Educação brasileira em conjunto com "altas habilidades", refere-se àqueles que possuem potencial elevado de forma isolada ou combinada nas áreas intelectual, acadêmica, liderança, psicomotricidade e artes, além de apresentar grande criatividade, envolvimento na aprendizagem e realização de tarefas em áreas de seu interesse. Estudantes com características de superdotação - ou talentosas - são considerados portadores de necessidades educacionais especiais (NEE), por serem detentores de traços individuais específicos que carecem de um acompanhamento singular (Brasil, 2008; Renzulli (2005). Neste contexto, a mentoria tanto contribui para a compreensão das variáveis que integram um contexto educacional promissor (Bisland, 2001) quanto se apresenta como uma das melhores formas de prover recursos a estudantes cujas necessidades especiais sejam de alto desempenho (Besnoy \& McDaniel, 2016).

A mentoria é uma modalidade de relação de apoio e suporte por parte de uma pessoa mais experiente que orienta, direciona e transfere conhecimento e experiência normalmente a uma pessoa mais jovem (Besnoy \& McDaniel, 2016; Shaughanessy, 2005; Zorman, Rachmel, \& Bashan, 2016). Ou seja, corresponde às leis e diretrizes da Política Nacional de Educação Especial na Perspectiva da Educação Inclusiva que tem como alvo atender às necessidades educacionais especiais de estudantes talentosos (Brasil, 2007) e contribuir para o processo de ensino-aprendizagem, identificação e desenvolvimento do potencial intelectual e criativo elevado destes estudantes. Outrossim, a educação regular ou tradicional não consegue suprir as exigências dessa população na criação de recursos, redes de apoio com a sociedade, empresas, profissionais de sucesso, laboratórios e outros ambientes que muito contribuem para a formação de mentores (Freeman, 1998).

No ambiente escolar, um dos relacionamentos de mentoria mais comuns e informais é o de um professor e um estudante, e esse professor tem a função de identificar e modelar habilidades diariamente a fim de encorajar a aprendizagem ao longo da vida, e não apenas para o momento (Davalos \& Haensly, 1997). Dessa forma, o estudante obtém de seus professores-mentores aconselhamento, direção e assistência. Outro tipo de relacionamento de mentoria é o de um aluno mais velho que orienta um aluno mais jovem. Ao emparelhar um estudante mais jovem com um estudante do Ensino Médio, por exemplo, ou o estudante do Ensino Médio com o universitário, o mais jovem terá um modelo para o sucesso. Ele vê o mais velho como alguém com o mesmo histórico de vida, um modelo a seguir. Ainda outro benefício proveniente dessa relação é o sentimento de responsabilidade do mais velho pelo mais novo (Grybek, 1997; Wright \& Borland, 1992).

É importante ter em mente que, ao serem estabelecidos os pares, as estratégias de mentoria devem ser claras e estruturadas de acordo com os objetivos propostos. Estes podem incluir um programa de leitura, provisão de feedback sistemático sobre o desempenho do mentoreado, o exercício de papéis como o de guia ao mostrar o caminho e os obstáculos do percurso na busca pelos objetivos, o papel de conselheiro ao discorrer sobre comportamentos inapropriados, de amigo oferecendo suporte emocional, além do papel de modelo como exemplo de valores, atitudes e padrões de comportamento (Kram, 1985; Rhodes, 2002).

Vale destacar que nem todas as pessoas ou profissionais são bons candidatos a serem mentores. Acima de tudo, o candidato a mentor deve possuir especialização no campo de interesse do mentoreado talentoso/superdotado e ser compromissado com o processo de mentoria. Considerando que não existe um perfil específico de mentor, a escola deve usar vários recursos e técnicas - como entrevistas e perfis - para 
escolher os mentores certos para seu programa. Isso porque é preciso, acima de tudo, responsabilidade e disposição para compartilhar conhecimento e assumir múltiplos papéis como o de professor, especialista, guia, conselheiro, amigo e modelo (Clasen \& Clasen, 1997; Dondero, 1997; Schatz, 1999).

Em países onde a mentoria faz parte do processo de formação do estudante, nem sempre o trabalho com os que são talentosos está dentro de instituições de ensino. Por vezes, ela ocorre em uma biblioteca de universidade acompanhada por um professor universitário, em um laboratório acompanhado por um supervisor (Subotnik, 1988).

Crianças e adolescentes que frequentam acampamentos de verão, organizados especialmente para superdotados em países como os EUA, por exemplo, frequentemente identificam-se com mentores os quais acabam se tornando referências valiosíssimas em sua vida (Durden \& Tangherlini, 1993) e costumam ser acompanhadas pela escola ou família a fim de terem resguardadas sua proteção no processo de mentoria. Percebe-se, portanto, que o impacto da ação do mentor pode ir além da escola, se encontra ainda em programas de extensão e atualização, treinamento e transmissão de conhecimento oral de geração em geração, aprendizagem e participação ativa (Delors, 1996; Perrenoud, 1999).

A origem do conceito "mentoria" se encontra na Grécia Antiga, no século VIII a.C., na obra-prima de Homero "Odisséia, as aventuras de Ulisses" (Homero, 1960). Nela, Ulisses, rei de Ítaca, sai de casa para lutar na Guerra de Tróia e deixa sua esposa Penélope e o filho Telêmaco. Ao amigo Mentor confia a educação do filho. Mentor passa a ser seu professor, mas também amigo, conselheiro e protetor ao contribuir para sua formação e introduzi-lo no mundo adulto (Grassinger, Porath, \& Ziegler, 2010). A narrativa impactou tanto a nobreza da época, que esta passou a buscar "mentores" para auxiliarem na educação de seus filhos (Bellodi, 2003).

$\mathrm{O}$ conceito de mentor tem sido confundido com outros, como preceptor, supervisor, tutor e coach, causando, por vezes, confusão e exigindo sua especificação (Botti \& Rego, 2008). Para Bragotto (2005), entretanto, independentemente da proximidade de significados, nenhuma expressão é suficiente para expressar seu real sentido. Atualmente e conforme já foi introduzido, a mentoria refere-se à relação entre duas ou mais pessoas, normalmente uma mais experiente, que proporciona orientação, treino, ensino, modelagem de papel, compartilhamento de contatos, redes de relacionamento e apoio em um campo selecionado (Besnoy \& McDaniel, 2016). O termo figura nos dicionários e na percepção de diferentes autores como uma grande variabilidade de sinônimos. Somente Crisp e Cruz (2009) encontraram mais de 500 deles em seu estudo de revisão bibliográfica, incluindo termos como protetor, atencioso, responsável pelo desenvolvimento físico, social, espiritual e intelectual dos mais jovens, mestre, guia etc.

O conceito de mentoria compreende ainda duas grandes funções: a psicossocial e a função de orientação profissional ou de carreira. A psicossocial está relacionada à construção de relacionamentos que transmitem confiança, intimidade e relação interpessoal significativa, a qual tende a melhorar a autoaprendizagem, autoeficiência e autoeficácia dos envolvidos. Ou seja, trata-se de elementos da relação que aprimoram o senso de competência, responsabilidade pessoal, clareza de identidade e efetividade no desempenho de qualquer que seja a atividade. São aspectos eminentemente comportamentais. A função de carreira ou orientação profissional diz respeito aos aspectos da relação que garante a aprendizagem dos papéis que o indivíduo assume ou assumirá profissionalmente e que prepara o mentoreado para o progresso acadêmico e profissional (Ragins \& Kram, 2007; Noe, 1988).

São vários os tipos de relação de mentoria. Há a relação formal ou informal formalizada e sistematizada, um a um, grupal, mista, de caráter voluntário, obrigatória, predeterminada ou determinada, ao vivo ou virtual, on-line e peer-mentoring. Há ainda as relações díades, bem pessoais entre um mentor profissional e o aprendiz, e ainda e-mentoring (a distância utilizando a internet), sem relação pessoal, oferecido por pessoas leigas, não treinadas pedagogicamente, mas que permite comunicação entre os participantes em sua conveniência e em diferentes fusos horários (Ragins \& Kram, 2007).

Percebe-se que a mentoria pode oferecer aos estudantes talentosos/superdotados oportunidades que vão ao encontro de suas necessidades de instrução, aprendizagem orientada para a melhoria com amplas possibilidades de prática e feedback direto (Grassinger et al., 2010). Outrossim promove desafios, amadurecimento, rigor acadêmico, exploração de carreiras futuras e satisfação pela possibilidade de aplicação do conhecimento adquirido (Templin, 1999). A relação mentor versus mentoreado contribui ainda para a interação de estudantes talentosos com adultos significativos, e/ou especialistas nas mais diversas áreas por meio de um atendimento face a face (Cakir \& Kocabas, 2016).

Para Berger (1990) e Gagné (2004), a exposição de um estudante talentoso/superdotado a um mentor disposto a compartilhar valores e experiências pessoais, interesses específicos, tempo e habilidades é uma das experiências mais valiosas que esta população pode obter. Muitos desses estudantes gostam de tudo e são realmente bons em várias áreas, mas podem ter problemas no planejamento de carreira caso não consigam estabelecer prioridades ou metas em longo prazo. 
Assim, quando a mentoria é estruturada adequadamente, o relacionamento beneficia o mentoreado, mas igualmente o mentor, fornecendo a ambos, incentivo, inspiração, novos insights e outras recompensas pessoais. Em outras palavras, abrem-se oportunidades para jovens estudiosos examinarem aspectos da integração da vida profissional com a vida pessoal. Tais experiências são cruciais para a tomada de decisão sobre a escolha de uma carreira em determinado campo de atividade.

A mentoria do talento/superdotação é ainda muito válida para populações específicas, como estudantes de origens desfavorecidas (Hicks \& Ranis, 2001) e alunos com desafios de aprendizagem (Shevitz, Weinfeld, \& Jeweler, 2003). Esses normalmente precisam de um encorajamento diferenciado e direções mais concretas a fim de que possam navegar em ambientes que não Ihes são familiares (Ming Liu \& Waller, 2018). Meninas, moças e mulheres talentosas constituem outro grupo de população específica cuja mentoria se faz necessária. Há, entre elas, as que possuem sentimentos de isolamento e marginalização em ambientes acadêmicos, ou são negligenciadas, deixadas de lado ou fora dos bastidores onde conversas informais são cruciais no direcionamento de ideias, indicações e/ou obtenção de mandatos. Nesse sentido, é preciso orientar meninas talentosas no processo de desenvolvimento de seu potencial em termos de realização e satisfação da vida (Hill, Corbett, \& Rose, 2010; Kerr \& Gahm, 2018).

Assim, a mentoria e seus programas são oportunidades de mostrar à sociedade e aos formuladores de políticas públicas formas de agregar valores diante de ocasiões reais de crescimento dos alunos (Subotnik, Olszewski-Kubilius, \& Worrell, 2018). Nesse sentido, é importante reconhecer e estimular em sala de aula um aprendizado voltado para a ampliação de um conceito que valoriza a responsabilidade, o espírito de equipe, a ética, o respeito, a cidadania e práticas educativas que desenvolvam a curiosidade, a capacidade criadora, a socialização e o raciocínio lógico do educando, entre outros aspectos. Para tanto, os estudantes devem ser imergidos em situações genuínas em que vão se tornando responsáveis pela produção de resultados específicos (Alencar \& Fleith, 2001; Maia-Pinto \& Fleith, 2002).

Diante dessa breve revisão da temática da mentoria de estudantes talentosos no contexto educacional regular, foi possível perceber que há inúmeras formas de aplicação dessa metodologia. A escola, no papel de seus gestores, não deve sacrificar o atendimento às necessidades educacionais de seus estudantes talentosos/superdotados enquanto depende unicamente do comprometimento dos responsáveis pela aplicabilidade de leis e diretrizes já estabelecidas pela Política Nacional de Educação Especial na Perspectiva da Educação Inclusiva (Brasil, 2008). Outrossim, não deve ficar apenas na dependência do estabelecimento de Núcleos de
Atividades de Altas Habilidades/Superdotação (NAAH/S) nas proximidades de sua escola, pois normalmente há apenas um em cada estado, sob a gerência da Secretaria de Educação Especial do Ministério de Educação. Essa situação justifica a escola regular - no papel de seus diretores, professores e demais agentes educacionais, além da comunidade - tomar a iniciativa de proporcionar condições para identificar o estudante talentoso/superdotado a fim de oferecer-lhe o atendimento necessário.

Percebe-se que é possível a escola, em acordo e parceria com todos os seus profissionais, a partir da definição e formação do perfil de um mentor, atuar com a população de estudantes talentosos/superdotados. O mentor poderia ser o próprio professor da turma, ou professores de níveis de ensino mais elevados, alunos mais velhos, profissionais das mais diversas áreas relativas aos interesses específicos desses estudantes, especialistas contratados e mesmo membros da comunidade dispostos a compartilhar seu conhecimento. Os mentores atuariam em parceria com os professores regentes a fim de, juntos, proverem mais e melhor atendimento às necessidades educacionais especiais desses estudantes no próprio ambiente escolar, como na biblioteca, no laboratório, na sala de estudo, ou mesmo nas salas de aulas que se encontram vazias em determinados horários, além de, conforme já foi dito, salas de recursos multifuncionais.

Atualmente, as metas de desenvolvimento de talentos apontam para a emergência de um novo paradigma que desafia educadores a assumirem o papel de catalisadores do potencial do estudante, a fim de contribuírem para seu melhor desenvolvimento (Oliveira, 2010). Nesse contexto, a capacitação do professor para a identificação do talento/superdotação é um dos mais importantes e consistentes recursos por ocuparem uma posição de extrema relevância em relação ao reconhecimento das capacidades elevadas (Araújo, 2011; Wechsler \& Souza, 2011).

Diante do objetivo desse estudo, que se refere à busca e análise de produção científica tanto nacional quanto internacional sobre mentoria do talento, foi realizada a pesquisa do "Estado da Arte" ou "Estado do Conhecimento", definida como de caráter bibliográfico cuja finalidade é mapear certas produções acadêmicas em diferentes campos do conhecimento (Severino, 1986). Para tanto, o estudo procurou apresentar os seguintes resultados de análise: ano de publicação, amostra, objetivos e conclusão, instrumentos, tipos de estudo e fonte de publicação, o que será analisado a seguir.

\section{MÉTODO}

Foi realizada uma revisão da literatura em estudos teóricos e empíricos sobre a temática da mentoria do talento e consultadas as seguintes bases de dados eletrônicas de publicações periódicas, teses e dissertações: 
Banco de Teses da Coordenação de Aperfeiçoamento de Pessoal de Nível Superior - Capes e Portal Periódicos Capes; Supporting Advanced and Gifted Education - SAGE; Scientific Electronic Library Online - SciELO; Education Resources Information Center - ERIC; base de dados multidisciplinar-Academic Search Primier e a plataforma de dados que contempla estudos na área de saúde ProQuest. Dessas bases, três destacaram-se: o Banco de Dissertações/ Teses-Capes, com 17 estudos, a SCIELO, com 15 estudos, e a SAGE Journals, com 13 estudos.

Considerando a necessidade de filtrar o número de artigos provenientes dos termos indicados, priorizaram-se os estudos entre os anos de 2000 e 2018. Quanto aos termos descritores em língua inglesa, utilizaram-se os seguintes: mentor, mentoring, mentorship, talented, gifted, high ability, Education, High School and High Education nas mais diversas combinações e diante de uma busca específica ou avançada. Já em português, priorizaram-se os seguintes termos: mentoria, mentoria do potencial, mentoria de estudantes talentosos, mentoria na educação, mentoria na escola, talento, altas habilidades, superdotação, Educação, Ensino Fundamental, Médio, Superior, e delimitaram-se ainda os seguintes tipos de pesquisa: qualitativa e quantitativa.

Após várias fases e delimitações, a fase semifinal resultou em 169 artigos, sendo 127 internacionais e 42 nacionais. Após uma leitura atenta desses textos, restaram 59 estudos entre artigos, teses e dissertações, sendo 35 internacionais e 24 nacionais. Estes foram selecionados por combinarem o contexto acadêmico (Ensino Infantil, Fundamental, Médio e Superior) e o estudo da mentoria do talento dentre centenas de outros que se referiam à temática, mas não cumpriam os requisitos exigidos.

Ressalta-se ainda que não foram encontrados estudos sobre a mentoria de estudantes talentosos nem superdotados no Brasil, mas somente a mentoria no contexto educacional geral, contrário aos estudos internacionais, os quais se referem, todos, à temática. Outro aspecto a considerar é que, mesmo entre as bases de dados do Brasil, foram utilizados descritores em inglês. O motivo foi a percepção de que alguns pesquisadores brasileiros preferem esses termos, como é o caso do estudo de Martins e Bellodi (2016), "Mentoring: uma vivência de humanização e desenvolvimento no curso médico", ou o estudo de Bellodi, Chebabo, Abensur e Martins (2011), "Mentoring: ir ou não ir, eis a questão: um estudo qualitativo".

\section{Ano de Publicação e Amostras}

Esta informação refere-se à amplitude do período de publicação dos estudos localizados. Foram encontrados, nos anos de 2010, 2011 e 2016, quatro estudos nacionais em cada. Entre os estudos internacionais, destaca-se o ano de 2014 com seis estudos, seguidos dos anos 2000, 2010 e 2011 com quatro estudos cada. Quanto às amostras dos estudos nacionais descritos na Tabela 1, foram encontradas 12 referências a professores da Educação Básica (Ensino Infantil, Fundamental I, II e Médio) e universidade, seguidos de nove estudos envolvendo estudantes universitários, especialmente de cursos de medicina. Quanto às amostras internacionais, elas estão mais bem distribuídas. As mais atuais se referiram a estudantes talentosos do Ensino Fundamental e Médio, a estudantes não talentosos do Ensino Médio,

Tabela 1. Amostra de participantes de estudos nacionais e internacionais sobre mentoria do talento.

\begin{tabular}{llc}
\hline \multicolumn{1}{c}{ Itens } & N & I \\
\hline Estudantes Ensino Fundamental & - & 3 \\
Professores Mentores (Especialistas) & - & 4 \\
Estudantes/Professores de Programa de Desenvolvimento de T/S & - & 2 \\
Professores do Ensino Fundamental, Médio e Universitários & 12 & 4 \\
Estudantes Talentosos (EF e EM) & - & 5 \\
Estudantes Não Talentosos do Ensino Médio & - & 5 \\
Registros de entrevistas arquivadas & 1 & 2 \\
Estudantes/Professores de escolas de idiomas & 1 & 1 \\
Estudantes Universitários & 9 & 4 \\
Estudantes Negros & - & 1 \\
Adultos T/Ss; Voluntários & - & 1 \\
Estudantes T/Ss em matemática (EF e EM) & - & 3 \\
Escritores “como mentores" & 1 & 35 \\
\hline Total & $\mathbf{2 4}$ & \\
\hline
\end{tabular}

Fonte: Elaborada pelos autores. N - Estudos Nacionais; I - Estudos Internacionais; T/S - Talentosos/Superdotados ou Talento/ Superdotação; EF - Ensino Fundamental; EM - Ensino Médio. 
a professores mentores, professores do Ensino Fundamental, Médio e a estudantes universitários. As demais amostragens incluem professores e estudantes de cursos de idiomas, escritores influenciados por mentores e análise de publicações e relatórios sobre mentoria, entre outros. Dentre os estudos que discorrem sobre a temática, destaca-se o de Mira e Romanowski (2016).

\section{Objetivos e Conclusões do Estudo}

Segundo a Tabela 2, entre alguns dos objetivos de estudos nacionais está incluída a investigação de estudos que se referem ao processo de formação de professores, compreensão da adesão de alunos a programas de mentoria e o interesse pela busca da percepção dos participantes sobre a experiência vivida como mentoreados, análise do perfil dos mentores e identificação das características de professores que vão além do ofício de ensinar. Dois estudos que exemplificam estes aspectos são os de Migliorança (2010) e de Ribeiro et al. (2013). Quanto aos objetivos de estudos internacionais, a maioria propõe: relatar experiências de estudantes do Ensino Médio e Superior como mentores de estudantes talentosos do Ensino Infantil e Fundamental; verificar a opinião desses estudantes sobre Programas de Mentoria; verificar se práticas de mentoria contribuem para o desenvolvimento do capital psicológico; e compreender a adesão dos alunos a um Programa de Mentoria.

Entre as conclusões de estudos nacionais, os quais analisam o processo de formação de professores por meio da mentoria em início de carreira, foram encontradas muitas expressões relacionadas a ansiedade, angústia, insegurança, desespero e falta de apoio por parte da escola. Por outro lado, o professor-mentor é visto como alguém que possui conhecimento, experiência, disponibilidade, relacionamento interpessoal, motivação e liderança, e, ainda, há referência ao fato de que há escassez de Programas de Mentoria direcionados ao professor. Conclusões de estudos internacionais, incluem, entre outros aspectos, benefícios da mentoria, habilidades de pesquisa, competência profissional e aumento linear do capital psicológico dado à mentoria. Ademais, verificou-se que estudos apontam o seguinte: alguns estudantes talentosos não se sentem mentoreados; outros não estão prontos ou não querem ter um mentor. De forma geral, entretanto, os estudos concluem que há benefícios psicossociais e de carreira, além da influência positiva de um adulto significativo.

\section{Instrumentos}

Conforme listado na Tabela 3, percebe-se que há instrumentos ou técnicas que se repetem tanto nos estudos nacionais quanto internacionais. São entrevistas, questionários, narrativas e diários on-line. Inúmeros estudos fazem uso de mais de um instrumento, razão da quantidade ser maior que o número de estudos. Estudos internacionais fazem uso de instrumentos mais específicos para a avaliação de estudantes talentosos, bem como uma maior variedade deles.

\section{Tipos de Estudo e Fontes de Publicação}

Não foram encontrados artigos nacionais de cunho experimental (quantitativo nem qualitativo) no nível da Educação Básica e que discorressem sobre a temática da mentoria do talento. Há, neste contexto, uma quantidade razoável de estudos de cunho bibliográfico, característica não considerada para seleção. Assim, dos estudos localizados foram selecionadas nove dissertações e cinco teses da base de dados "Banco de Dissertações e Teses (Capes) que se relacionam com a mentoria no contexto educacional brasileiro. Portanto, de forma geral, quanto ao tipo de estudos, sobressaíram-se os qualitativos em $1 .^{\circ}$ lugar, e em $2 .^{\circ}$, quantitativos/qualitativos.

Quanto às fontes de publicação internacionais, percebeu-se que a temática é de interesse especial das áreas de educação e psicologia. A despeito dessa variabilidade, destacaram-se fontes, como: Journal for the Education of the gifted, com seis publicações, e a Roeper Review, com cinco. Outras duas fontes de publicação foram tidas como sendo promissoras na pesquisa da temática: Journal of Advanced Academics e Thinking Skills and creativity-Elsevier. O maior número de fontes de publicações nacionais está nos Banco de Dissertações e Teses Capes, com um total de 14, com destaque para as universidades Ufscar, de São Carlos, e a Faculdade de Boa Viagem, Recife. Ou seja, muitos estudos da área de mentoria ligada à educação não têm sido publicados para um grupo maior de pesquisadores por meio de artigos. Em relação aos demais, aqueles que são publicados encontram-se em fontes como: Revista de Psicologia; Revista Educação e Pesquisa; Revista Interface de Botucatu (on-line); Revista de Educação Médica; Reflexão e Ação e Comunicação; e Saúde e Educação.

\section{CONSIDERAÇÕES FINAIS}

O levantamento das pesquisas sobre a mentoria de estudantes talentosos demonstrou a necessidade de considerar uma ampla variedade de termos a fim de chegar aos dois constructos finais, a saber, "mentoria" e "talento", ou, ainda, "superdotação". Foi possível compreender que este tema está mais presente na literatura inglesa comparada à brasileira. A presença da temática da mentoria e não mentoria do talento no contexto educacional se faz presente não apenas no Ensino Fundamental e Médio, mas se estende a outros níveis e áreas, com ênfase inclusive nos cursos de inglês e área médica. Na educação, há ênfase na formação do professor em início de carreira. Quanto à ausência de instrumentos psicológicos nacionais, padronizados e validados para uso de psicólogos escolares na identificação dessa população, reflete-se a percepção de Güenther (2003) ao afirmar que os professores não são instruídos 
Tabela 2. Amostra de áreas, objetivos e conclusões de estudos Nacionais e Internacionais.

\begin{tabular}{|c|c|c|c|c|}
\hline Áreas & Objetivos & Conclusões & $\mathbf{N}$ & $\mathbf{I}$ \\
\hline $\begin{array}{l}\text { E-Mentoring; } \\
\text { DVC }\end{array}$ & $\begin{array}{l}\text { Investigar o papel de e-mentoring; tecnologia de } \\
\text { Videoconferência de Desktop (DVC) e PM on-line } \\
\text { entre professores e T/S; }\end{array}$ & $\begin{array}{l}\text { Espaço de interação com T/S; motivação } \\
\text { e perseverança em tarefas individuais e } \\
\text { grupais; intervenções positivas de DVC; }\end{array}$ & 4 & 3 \\
\hline $\begin{array}{l}\text { Mentoria e } \\
\text { criatividade }\end{array}$ & $\begin{array}{l}\text { Analisar as estratégias de mentoria para o ensino } \\
\text { de pensamento criativo; investigar as crenças e } \\
\text { influência de mentores sobre alunos criativos; }\end{array}$ & $\begin{array}{l}\text { Estratégias de mentoria são eficazes para } \\
\text { T/S e não T/S; há crenças de professores e } \\
\text { mães sobre criatividade; }\end{array}$ & & 3 \\
\hline $\begin{array}{l}\text { Mentoria } \\
\text { em minorias } \\
\text { talentosas }\end{array}$ & $\begin{array}{l}\text { Firmar um compromisso com pesquisa científica } \\
\text { por meio de PM para T/Ss de minoria étnica; } \\
\text { acompanhar as minorias no desenvolvimento de } \\
\text { identidade matemática social, racial e de gênero; }\end{array}$ & $\begin{array}{l}\text { Programas de enriquecimento têm impacto } \\
\text { entre minorias; há desafios internos que os } \\
\text { adolescentes negros procuravam negociar } \\
\text { com êxito dentro e fora da escola; }\end{array}$ & & 2 \\
\hline $\begin{array}{l}\text { Efeito de } \\
\text { programas de } \\
\text { mentoria em } \\
\text { T/SD; }\end{array}$ & $\begin{array}{l}\text { Investigar a provisão educacional para estudantes } \\
\text { T/S em matemática; examinar impacto do Projeto } \\
\text { M3 e currículo de mentoria; verificar os efeitos } \\
\text { de programas de mentoria no desempenho } \\
\text { acadêmico de estudantes T/Ss; }\end{array}$ & $\begin{array}{l}\text { Aquisição de conhecimento ao participar } \\
\text { do PM; aspectos positivos/negativos em } \\
\text { programas; significativa compreensão de } \\
\text { conceitos matemáticos; não encontrado } \\
\text { efeito no desempenho acadêmico; }\end{array}$ & & 7 \\
\hline $\begin{array}{l}\text { Desempenho } \\
\text { de meninas } \\
\text { nas áreas } \\
\text { STEM }\end{array}$ & $\begin{array}{l}\text { Verificar se PMs ajudam a melhorar baixo } \\
\text { desempenho de meninas nas áreas STEM; testar } \\
\text { unidades curriculares do projeto M3 para alunas } \\
\text { T/Ss; construir confiança matemática; analisar } \\
\text { percepção de pais sobre o talento matemático; }\end{array}$ & $\begin{array}{l}\text { Ganhos significativos em participantes } \\
\text { de PM; esses aumentam a confiança e } \\
\text { competência em matemática; não houve } \\
\text { efeito significativo sobre o desempenho } \\
\text { acadêmico e autoeficácia; }\end{array}$ & & 5 \\
\hline $\begin{array}{l}\text { Programa de } \\
\text { Mentoria de } \\
\text { Verão }\end{array}$ & $\begin{array}{l}\text { Examinar experiências de adolescentes T/Ss } \\
\text { que frequentam PM universitário de verão; } \\
\text { percepções de si e suas relações com mentores; }\end{array}$ & $\begin{array}{l}\text { Aumento nas habilidades de pesquisa e } \\
\text { competência profissional; relacionamentos } \\
\text { positivos com mentores (tempo / } \\
\text { acessibilidade/); }\end{array}$ & & 2 \\
\hline $\begin{array}{l}\text { Formação de } \\
\text { líderes pela } \\
\text { mentoria }\end{array}$ & $\begin{array}{l}\text { Cultivar líderes em várias áreas por meio de } \\
\text { PM; investigar o conhecimento de conselheiros } \\
\text { escolares sobre T/S; }\end{array}$ & $\begin{array}{l}\text { Há o desejo de restabelecer relação } \\
\text { de mestre e discípulo; pode ocorrer de } \\
\text { transformar e ser transformado; }\end{array}$ & & 5 \\
\hline Revisão & $\begin{array}{l}\text { Revisar bibliografia de PM em universidades; } \\
\text { analisar registros de entrevistas de estudantes T/S } \\
\text { que participaram de PM; }\end{array}$ & $\begin{array}{l}\text { Experiência positiva de PM; fragilidades } \\
\text { metodológicas que dificultam obtenção de } \\
\text { conclusões sólidas sobre a eficácia de PM; }\end{array}$ & 1 & 1 \\
\hline $\begin{array}{l}\text { Mentoria de } \\
\text { escritores }\end{array}$ & $\begin{array}{l}\text { Investigar o perfil dos mentores e sua influência } \\
\text { na produção literária; }\end{array}$ & $\begin{array}{l}\text { Competência profissional, conhecimento } \\
\text { técnico, criatividade, valores éticos, } \\
\text { satisfação; }\end{array}$ & 1 & \\
\hline $\begin{array}{l}\text { Mentoria e } \\
\text { formação de } \\
\text { professores }\end{array}$ & $\begin{array}{l}\text { Analisar o processo de formação em início de } \\
\text { carreira; investigar contribuição da prática de } \\
\text { mentoria; definir finalidades e orientações } \\
\text { metodológicas de um PM; analisar contribuições } \\
\text { de mentores no processo de inserção docente; } \\
\text { Identificar influência de PM na formação do } \\
\text { professor; }\end{array}$ & $\begin{array}{l}\text { Mentores com conhecimento, mas sem } \\
\text { evidência de identidade com T/Ss; início } \\
\text { da carreira caracterizado por insegurança } \\
\text { e falta de apoio; professor-mentor mais } \\
\text { bem preparado; melhor relacionamento } \\
\text { interpessoal; poucos PMs para professores; }\end{array}$ & 11 & \\
\hline $\begin{array}{l}\text { Mentoria } \\
\text { para } \\
\text { estudantes } \\
\text { de diversos } \\
\text { níveis }\end{array}$ & $\begin{array}{l}\text { Relatar experiências de estudantes do EM e } \\
\text { ES como mentores de T/Ss do El e EF; verificar } \\
\text { opinião de T/Ss sobre PM com a universidade; } \\
\text { analisar se práticas de mentoria contribuem para } \\
\text { o desenvolvimento psicológico do estudante T/S; } \\
\text { compreender a adesão dos alunos a um PM. }\end{array}$ & $\begin{array}{l}\text { Mais habilidades de pesquisa; } \\
\text { relacionamentos positivos com mentores; } \\
\text { há T/Ss que não se sentem mentoreados; } \\
\text { há quem não queira ter mentor; } \\
\text { benefícios psicossociais/carreira; melhor } \\
\text { capital psicológico; influência de adultos } \\
\text { significativos. }\end{array}$ & 1 & \\
\hline
\end{tabular}

Total

Fonte: Elaborada pelos autores: N - Estudos Nacionais; I - Estudos Internacionais; T/S - Talentosos/Superdotados; EF - Ensino Fundamental; EM - Ensino Médio; ES - Ensino Superior; Projeto M3 - Mentoring Mathematical Minds; PM - Programa de Mentoria; STEM - Science, Technology, Engineering and Mathematics. 
Tabela 3. Quantidade e Instrumentos presentes nos estudos Nacionais e Internacionais.

\begin{tabular}{|c|c|c|c|c|c|}
\hline \multicolumn{2}{|l|}{ Nacionais } & \multicolumn{4}{|c|}{ Internacionais } \\
\hline Diários on-line/ Campo & 1 & Questionários & 7 & Arquivos e base de dados & 3 \\
\hline Questionários & 9 & $\begin{array}{l}\text { Mathematical confidence and } \\
\text { skills }\end{array}$ & 1 & $\begin{array}{l}\text { Multiple Intelligences Assessment } \\
\text { Technique }\end{array}$ & 1 \\
\hline Entrevistas & 6 & $\begin{array}{l}\text { The National Mentoring } \\
\text { Program; }\end{array}$ & 1 & $\begin{array}{l}\text { Raven, Standard Progressive } \\
\text { Matrices Test }\end{array}$ & 1 \\
\hline $\begin{array}{l}\text { Modelo descritivo de } \\
\text { autoconhecimento }\end{array}$ & 1 & Entrevistas & 10 & $\begin{array}{l}\text { Mathematically promising English } \\
\text { language learners }\end{array}$ & 1 \\
\hline Gravações & 1 & Boletim Escolar & 1 & Videoconferência de Desktop (DVC) & 1 \\
\hline Análise documental & 2 & $\begin{array}{l}\text { Diários; correspondência; } \\
\text { diálogos on-line }\end{array}$ & 6 & $\begin{array}{l}\text { Project Support to Affirm Rising } \\
\text { Talent (START) }\end{array}$ & 1 \\
\hline Narrativas on-line 4 & 4 & $\begin{array}{l}\text { Torrance Test of Creative } \\
\text { Thinking (TTCT) }\end{array}$ & 3 & Projeto CLUE; Mentor Log & 1 \\
\hline $\begin{array}{l}\text { Programa de Formação de } \\
\text { Mentores - Ufscar }\end{array}$ & 3 & $\begin{array}{l}\text { The School's English } \\
\text { Proficiency Test }\end{array}$ & 1 & Programa GO-GIRL & 1 \\
\hline $\begin{array}{l}\text { Relatórios de participantes } \\
\text { de PM }\end{array}$ & 2 & $\begin{array}{l}\text { Motivated Strategies for } \\
\text { Learning Questionnaire }\end{array}$ & 1 & $\begin{array}{l}\text { Youth-Mentor Relation-ship } \\
\text { Quality Inventory }\end{array}$ & 1 \\
\hline Nominação por profs. & 1 & $M^{3}$ Mentoring/Math/Minds & 1 & WISC & 1 \\
\hline \multirow[t]{6}{*}{ Filmagens } & 1 & $\begin{array}{l}\text { Self-efficacy Perception Profile } \\
\text { for Adolescents }\end{array}$ & 2 & $\begin{array}{l}\text { Anotações de observ/ narrat/ } \\
\text { sistemáticas; }\end{array}$ & 1 \\
\hline & & $\begin{array}{l}\text { Teste de habilidade não verbal } \\
\text { de Naglieri; }\end{array}$ & 1 & $\begin{array}{l}\text { Classroom Instructional Practices } \\
\text { Scale }\end{array}$ & 1 \\
\hline & & Profile for Adolescents (SPPA) & 2 & Classroom performance & 1 \\
\hline & & lowa Test of Basic Skills & 4 & Mentoring Curriculum & 1 \\
\hline & & $\begin{array}{l}\text { Teste de Estimação e Avaliacão } \\
\text { de Resposta }\end{array}$ & 1 & Family Outreach Program & 1 \\
\hline & & $\begin{array}{l}\text { Mathematics Scales for Rating } \\
\text { the Behavioral Charact/of } \\
\text { Superior/Stud }\end{array}$ & 1 & Research Skills Questionnaire (RSQ & 1 \\
\hline Total & 31 & & & & 61 \\
\hline
\end{tabular}

Fonte: Elaborada pelos autores. PM - Programa de Mentoria.

nem instrumentalizados para trabalhar com essa população. Entretanto, quando devidamente preparados, são efetivos nas nomeações realizadas. Essa falta de instrumentos dificulta estabelecer uma cultura de atendimento à população de estudantes que se sobressaem por seu potencial, perdendo, assim, oportunidades de trabalhar potenciais especiais que seriam inclusive de benefício para a própria nação.

Quanto às limitações deste estudo, há de se relatar - especialmente sobre as bases de dados internacionais - a dificuldade de acesso a elas. Em muitos casos, o acesso só foi permitido nas próprias instituições, ou ainda, pagando pelo artigo. Desse modo, houve limitações na busca de dados devido à dificuldade de recuperação dos periódicos, ou seja, os dados aqui apresentados estão mais relacionados às possibilidades de acesso das pesquisadoras aos estudos do que propriamente uma quantidade existente no período de busca. É também importante considerar o fato de que muitas revistas científicas só passaram a integrar bases eletrônicas mais recentemente. Sendo assim, o fato de terem sido consultados somente bancos de dados eletrônicos precisa ser levado em consideração.

Como hipótese inicial, considerou-se a possibilidade de serem encontrados poucos estudos sobre mentoria no Brasil. Esta hipótese, entretanto, seria verdadeira se forem considerados apenas artigos. Na busca por dissertações e teses, tanto nas bases já pesquisadas quanto nas bibliotecas digitais de cada universidade, a impressão de "poucos" se dissipa, ou seja, a despeito de perceber que não se trata de uma temática comum no contexto nacional, alguns estudos significativos para a área existem, porém precisam apenas "sair" das dissertações e teses e virem para um campo de maior visibilidade. Nessa perspectiva, entre as sugestões para futuros estudos, deveria ser incluído um levantamento 
específico, abrangente e detalhado sobre o que tem sido estudado nos programas de Mestrado e Doutorado no Brasil.

\section{REFERÊNCIAS}

Alencar, E. M. L. S.; Fleith, D. S. (2001). Superdotação: determinantes, educação e ajustamento. São Paulo: EPU.

Araújo, M. R. (2011). Identificação e encaminhamento de alunos com indicadores de altas habilidades/superdotação na escola pública do município de fortaleza: proposta para a atuação de professores do atendimento educacional especializado. Dissertação de Mestrado, Faculdade de Educação, Universidade Federal do Ceará, Fortaleza.

Bellodi, P. L. (2003). O que é um tutor? Representações do papel em um grupo de professores de medicina durante $o$ processo de seleção. Revista Brasileira de Educação Médica 27(3), 205-212.

Bellodi, P. L.; Chebabo, R.; Abensur, S. I.; Martins, M. A. (2011) Mentoring: ir ou não ir, eis a questão: um estudo qualitativo. Revista Brasileira de Educação Médica, 35(2), 237-245. http://dx.doi.org/10.1590/S010055022011000200013

Berger, K. S. (1990). Mentor relationships and gifted learners (Report n. ${ }^{\circ}$ EDO-ED-90-5). Washington, DC: Office of Educational Research and Improvement.

Besnoy, K. D.; McDaniel, S. C. (2016). Going up in dreams and esteem: Cross-age mentoring to promote leadership skills in high school-age gifted students. Gifted Child Today. 39(1), 18-30. https://doi.org/10.1177/1076217515613386

Bisland. A (2001). Mentoring: An educational alternative for gifted students. Gifted Child Today, 24(4), 22-25. https:// doi.org/10.4219/gct-2001-550

Botti, S. H. O.; Rego, S. (2008). Preceptor, supervisor, tutor e mentor: quais são seus papéis?. Revista Brasileira de Educação Médica, 32(3), 363-373. http://dx.doi. org/10.1590/S0100-55022008000300011

Bragotto, D. (2005). O perfil e a influência do mentor na produção de escritores. Tese de Doutorado, Pontifícia Católica de Campinas, Campinas, SP.

Brasil. (2007). Política Nacional de Educação Inclusiva na perspectiva da Educação Inclusiva. Brasília: Ministério da Educação e do Desporto - SEE. In Inclusão: Revista da Educação Especial / Secretaria de Educação Especial. 1(1), p. 7-17, jan./jun. 2005. Recuperado em 3 fevereiro, 2018, http://portal.mec.gov.br/seesp/arquivos/pdf/ revinclusao5.pdf

Brasil. (2008). Política nacional de educação especial na perspectiva da educação inclusiva Brasília, DF: Grupo de Trabalho da Política Nacional de Educação Especial. Recuperado em 2 de março de 2017 de http://portal.mec. gov.br/arquivos/pdf/politicaeducespecial.pdf

Cakir, L.; Kocabas, I. (2016). Mentoring in Gifted Student's Education and a Model Suggestion. Educational Process: International Journal, 5(1), 76-90. https://doi. org/10.12973/edupij.2016.51.6
Clasen, J.; Clasen, J. (1997). Is mentoring necessary? Training and Development Journal, 39(4), 36-39.

Crisp, G.; Cruz, I. (2009). Mentoring college students: a critical review of the literature between 1990 and 2007. Research in Higher Education, 50, 525-545. https://doi. org/10.1007/s11162-009-9130-2

Davalos, R. A.; Haensly P. A. (1997). After the dust has settled: Youth reflect on their high school mentored research experience. Roeper Review, 19, 204-207.

Dondero, G. M. (1997). Mentors: Beacons of hope. Adolescence, $32,881-886$.

Durden, W.G., \& Tangherlini, A.E. (1993). Smart Kids: How Academic Talents are Developed and Nurtured in America. Toronto: Hogrefe and Huber.

Freeman, J. (2001). 'Mentoring gifted pupils'. Educating Able Children, 5, 6-12. Recuperado em 25 de março de 2018, de http://www.joanfreeman.com/pdf/Mentoring_Educ_ Able_01.pdf

Gagné, F. (2004). Transforming gifts into talents: The DMGT as a developmental theory. High Ability Studies, 15, 119-147.

Gagné, F.; Guenther, Z. C. (2012). Desenvolvendo talentos: Modelo Diferenciado de Dotação e Talento - DMGT 2.0. In Moreira, L. C.; Stoltz, L. (Eds.), Altas habilidades/ superdotação, talento, dotação e educação (pp. 20-61). Curitiba: Editora Juruá.

Grassinger, R.; Porath, M.; Ziegler, A. (2010). Mentoring the Gifted: A Conceptual Analysis. High Ability Studies, 21(1), 27-46. https://doi.org/10.1080/13598139.2010.488087

Grybek, D. D. (1997). Mentoring the gifted and talented. Preventing School Failure, 41(3), 115-118.

Guenther, Z. C. (2003). O aluno bem-dotado na escola regular: celebrando a diversidade, incluindo as diferenças. Revista Escritos sobre Educação, Ibirité, 2(1), 43-54.

Hicks, L.; Ranis. S. H. (2001). The Rosen scholars program: A new design for mentoring disadvantaged youth for postsecondary success. Evaluation summary (ED Publication No. 451789). https://eric.ed.gov/?id=ED451789

Hill, C.; Corbett, C. S. T.; Rose, A. (2010). Why so few? Women in science, technology, engineering, and mathematics. Washington, DC: American Association of University Women. Recuperado em 15 de fevereiro de 2018, de http:// www.aauw.org/files/ 2013/02/Why-So-Few-Women-inScience-Technology-Engineering-and-Mathematics.pdf

Homero. (1960). Ilíada (Nunes, C. A., Trad.). São Paulo: Melhoramentos.

Kerr, B. A.; Gahm, J. (2018). Developing talents in girls and Young women. In Pfeiffer, S. I.; Schunessy-Dedrick, E.; Foley-Nicpon, M. (Eds.), APA Handbook of giftedness and Talent (pp. 399-415). Washington, DC: APA.

Kram, K. E. (1985). Mentoring at work: developmental relationships in organizational life. Glenview, III: Scott, Foresman. 
Maia-Pinto, R. R.; Fleith, D. S. (2002). Percepção de professores sobre alunos superdotados. Estudos de Psicologia, 19(1), 78-90. http://dx.doi.org/10.1590/S0103$166 \times 2002000100007$.

Martins, A. E.; Bellodi, P. L. (2016). Mentoring: uma vivência de humanização e desenvolvimento no curso médico. Interface: Comunicação, Saúde, Educação, 20(58), 715-726. https://doi.org/10.1590/1807-57622015.0432

Migliorança, F. (2010). Programa de mentoria da UFSCAR e desenvolvimento profissional de três professoras iniciantes / Fernanda Migliorança. São Carlos: UFSCAR. 347p.

Ming Liu, W.; Waller, L. (2018). Identifying and educating underrepresented gifted students. In Pfeiffer, S. I.; Schunessy-Dedrick, E.; Foley-Nicpon, M. (Eds.), APA Handbook of giftedness and Talent (pp. 417-431). Washington, DC: American Psychological Association.

Mira, M. M.; Romanowski, J. P. (2016). Limites e possibilidades da atuação de mentores nos processos de inserção profissional docente. Revista Transmutare, 1(2),179-195. doi: $10.3895 /$ rtr.v1n2.4696.

Noe, R. A. (1988). An investigation of the determinants of successful assigned mentoring relationships. Personnel Psychology, 41, 457-479. https://doi. org/10.1111/j.1744-6570.1988.tb00638.x

Oliveira, C. T. (2010). Estratégia de aprendizagem e subjetividade em estudantes criativos do ensino superior. Dissertação de Mestrado em Educação, Curso de PósGraduação em Educação, Universidade de Brasília, Brasília, DF.

Payne, D.; Heilbronner, N. (2009). Mentoring talented science students: knowing the options. Gifted Education International, 25, 259-269. https://doi. org/10.1177/026142940902500307

Ragins, B. R.; Kram, K. E. (Eds.). (2007). The Handbook of Mentoring at Work: Theory, Research, and Practice. Thousand Oaks, CA: Sage.

Renzulli, J. S. (2005). The Three-Ring Conception of Giftedness: A Developmental Model for Promoting Creative Productivity. In Sternberg, R. J.; Davidson, J. E. (Eds.), Conceptions of giftedness (p. 246-279). Cambridge University Press. https://doi.org/10.1017/CBO9780511610455.015

Rhodes, J. E. (2002). Stand by me: The risks and rewards of mentoring today's youth. Cambridge, MA: Harvard University Press.
Ribeiro, M. M. F., Martins, A. F., Fidelis, G. T. A., Goulart, G. C., Molinari, L. C., Tavares, E. C. (2013). Tutoria em escola médica: avaliação por discentes após seu término e ao final do curso. Revista Brasileira de Educação Médica, 37(4), 509-514. https://dx.doi.org/10.1590/S010055022013000400006

Schatz, E. (1999). Matchmaking for young people. Journal of Secondary Gifted Education, 11, 67-86.

Severino, A. (1986). Metodologia do trabalho científico. São Paulo: Cortez \& Moraes.

Shaughnessy, M. F. (2005). The dynamic synergy between mentoring, nurturing and counseling the gifted. Gifted Education International, 19, 253-257. https://doi.org/10. 1177/026142940501900308.

Shevitz, B.; Weinfeld, R.; Jeweler S. (2003). Mentoring empowers gifted/learning disabled students to soar! Roeper Review, 26(1), 37-40. https://doi.org/10.1080/027831903095 54237

Subotnik, R. (1988). The motivation to experiment: A study of gifted adolescents' attitudes toward scientific research. Jour. for the Educ. of the Gifted, 11(3), 19-35. https://doi. org/10.1177/016235328801100303

Subotnik, R. F.; Olszewski-Kubilius, P.; Worrell, F. C. (2018). Talent development as the most promising focus of giftedness and gifted education. In Pfeiffer, S. I.; SchunessyDedrick, E.; Foley-Nicpon, M. (Eds.), APA Handbook of giftedness and Talent (pp. 231-245). Washington, DC: American Psychological Association.

Templin, M. A. (1999). A locally based science mentorship program for high achieving students: Unearthing issues that influence affective outcomes. School Science and Mathematics, 99, 205-212. https://doi. org/10.1111/j.1949-8594.1999.tb17475.x

Tourón, J. F.; Peralta, E. C.; Repáraz, C. (1998). La superdotación intelectual: modelos, identificación y estrategias educativas. Navarra: EUNSA.

Zorman, R.; Rachmel, S.; Bashan, Z. (2016). The national mentoring program in Israel - Challenges and achievements. Gifted Education International, 32(2) 173-184. https://doi. org/10.1177/026142941456066

Wechsler, S. M.; Souza, V. L. T. (2011). Criatividade e aprendizagem. Caminhos e descobertas em perspectiva internacional. São Paulo: Edições Loyola.

Wright, L.; Borland, J. H. (1992). A special friend: Adolescent mentors for young, economically disadvantaged, potentially gifted students. Roeper Review, 14, 124-129.

Reconhecimento ao apoio financeiro proveniente do Conselho Nacional de Desenvolvimento Científico e Tecnológico - CNPq

Recebido: 26 de agosto de 2018

Aprovado: 28 de março de 2019 